\title{
Índice verde $y$ disponibilidad de parques en zonas altamente densificadas de la Ciudad de México 2017-2020
}

\author{
Yazmín de las Nieves Téllez Montes \\ yaztem@gamail.com \\ https://orcid.org/0000-0002-9909-6289 \\ Instituto Politécnico Nacional \\ CIIEMAD-IPN-CIUDAD DE MÉXICO
}

Roque Juan Carrasco Aquino roquej1608@hotmail.com https://orcid.org/0000-0002-0988-0724 Instituto Politécnico Nacional CIIEMAD-IPN-CIUDAD DE MÉXIC0

José Teodoro Silva García tsilva09@hotmail.com Instituto Politécnico Nacional. CIIDIR-IPN-MICHOACÁN.

\section{Luis Fernando Ceja Torres lfceja@ipn.mx Instituto Politécnico Nacional. CIIDIR-IPN-MICHOACÁN}

Rodolfo Figueroa Brito rodofiguer@hotmail.com Instituto Politécnico Nacional. CEPROBI-IPN-MORELOS

Javier Pérez Corona jpcorona88@hotmail.com Instituto Politécnico Nacional. ESIA-UZ-IPN-CIUDAD DE MÉXICO

\section{RESUMEN}

La presente investigación tuvo como objetivo conocer el índice verde y la disponibilidad de parques en las alcaldías de mayor densidad poblacional en la Ciudad de México. Fue realizado mediante un análisis cuantitativo sobre bases de datos poblacionales proporcionados por el Instituto Nacional de Estadística y Geografía (INEGI) y Consejo Nacional de Población (CONAPO) para 2017 y 2020. El índice verde y la disponibilidad se calcularon mediante el geoprocesamiento de las áreas verdes correspondientes a parques de acuerdo con el Levantamiento de Áreas Verdes del 2017, considerando el portal de datos abiertos del gobierno de la Ciudad de México. Los datos geoestadísticos, 
fueron ajustados mediante la agrupación de polígonos y recálculo de áreas, no modificando la vectorización original. Los resultados indican que la alcaldía de Iztapalapa es la que mayor disponibilidad de parques tiene (259) y el mayor índice verde de áreas verdes en parques con un valor de $1.64 \mathrm{~m}^{2} \mathrm{hab}^{-1}$. Iztacalco, que es la alcaldía con mayor densidad de población registra un índice de $0.2 \mathrm{~m}^{2} \mathrm{hab}^{-1}$. Lo anterior refleja la enorme necesidad que existe de abrir espacios verdes recreativos a efecto de equilibrar la densidad de población con su índice verde en parques.

Palabras clave: parques, SIG, planeación urbana, índice verde, sustentabilidad 


\title{
Green index and availability of parks in highly densified areas of Mexico City 2017-2020
}

\begin{abstract}
The objective of this research was to know the green index and the availability of parks in the demarcations with the highest population density in Mexico City. It was carried out through a quantitative analysis on population databases provided by National Institute of Statistic and Geography (INEGI) and National Population Council (CONAPO) for 2017 and 2020. The green index and availability were calculated by geoprocessing the green areas corresponding to parks in accordance with the 2017 Green Areas Survey, considering the open data portal of the government of Mexico City. Geostatistical data was adjusted by grouping polygons and recalculating areas, without modifying the original vectorization. The results indicate that the demarcation of Iztapalapa is the one with the highest availability of parks (259) and the highest green index of green areas in parks with a value of $1.64 \mathrm{~m}^{2} \mathrm{hab}^{-1}$. Iztacalco, which is the demarcation with the highest population density, registers an index of $0.2 \mathrm{~m}^{2} \mathrm{hab}^{-1}$. This reflects the enormous need that exists to open recreational green spaces to balance the population density with its green index in parks.
\end{abstract}

Keywords: parks, GIS, urban planning, green index, sustainability

Artículo recibido: 05 de Mayo 2021 Aceptado para publicación: 20 de Junio 2021

Correspondencia: yaztem@gmail.com

Conflictos de Interés: Ninguna que declarar 


\section{INTRODUCCIÓN}

La Ciudad de México, (CDMX), antes conocida como Distrito Federal, al ser el principal centro político y económico del país y gracias al fenómeno de la "gentrificación", actualmente cuenta con una población de 9,209,944 habitantes (Instituto Nacional de Geografía y Estadística, 2020), distribuidos en una superficie de aproximadamente 1,494 $\mathrm{km}^{2}$, equivalente al $0.08 \%$ de la superficie del territorio mexicano siendo la densidad de

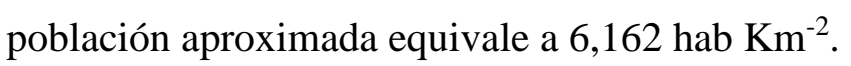

Es importante considerar la densidad de población como una de las variables fundamentales para la planeación urbana, toda vez que las zonas con mayor concentración presentarán un mayor reto al tener mayor demanda de servicios urbanos entre los que se incluyen los servicios ecosistémicos (PAOT, 2010).

La manera desorganizada del crecimiento urbano en la CDMX ha generado una infinidad de problemas de convivencia social entre los que destacan la compleja movilidad, un crecimiento exponencial de vivienda e inseguridad, por mencionar algunos, ocasionados por la falta de verdaderos esquemas de planificación urbana (Gobierno de la Ciudad de México, 2019). En este entorno, se agrega la nula importancia que se le ha dado al estudio, planeación y ordenación del territorio a los parques, que son áreas verdes de interacción directa con la naturaleza, espacios de acceso público que generan múltiples beneficios a la sociedad y medio ambiente.

Una particularidad en el caso del estudio de parques públicos es que la simple determinación mediante el análisis de imágenes satelitales es incapaz de distinguir los espacios públicos de los privados, y tomar en cuenta los diferentes usos del suelo. Para esto, se deben referir las imágenes a bases de datos espaciales que contengan esta información.

Estos enfoques cuantitativos se han utilizado en todo el mundo para cuantificar el índice verde en diferentes comunidades urbanas y más recientemente la accesibilidad. En 2010, se obtuvo el índice verde de tres comunas de la zona metropolitana de la ciudad de Santiago de Chile: La Pitana, $\left(2.4 \mathrm{~m}^{2} \mathrm{hab}^{-1}\right)$, San Miguel $\left(2.7 \mathrm{~m}^{2} \mathrm{hab}^{-1}\right)$ y Vitacura (56 $\mathrm{m}^{2} \mathrm{hab}^{-1}$ ) (Reyes Paecke \& Figueroa, 2010). Un estudio en distintos distritos urbanos españoles propone un índice ponderado que mide las áreas verdes urbanas disponibles en muros y azoteas utilizando una combinación de imágenes de satélite y trabajo de campo 
y las actitudes de los residentes a tener y cuidar jardines urbanos. (Briz de Felipe \& Felipe, 2017).

Dos estudios realizados en Argentina, el primero, en la ciudad de Rio Cuarto, estimó una disponibilidad promedio de $28.5 \mathrm{~m}^{2} \mathrm{hab}^{-1}$ (Galfioni, Degioanni, \& Maldonado , 2017), el segundo, analiza la superficie de los espacios verdes públicos del municipio de Santa Fe y los relaciona con cuatro áreas de calidad de vida. Determinando finalmente que este municipio cuenta con una superficie de espacios verdes públicos de $9.2 \mathrm{~m}^{2} \mathrm{hab}^{-1}$, para una población al 2010 de 390,745 habitantes (Gómez \& Velázquez, 2018). Para el año de 2019 en Colombia, se presenta una metodología para aplicar radios de acceso de 3,000 metros para parques y 400 metros para plazas.

Adicionalmente se calculó el índice verde para la comunidad del Gran Santiago, concluyendo que solo el $5.7 \%$ de la población cumple con la recomendación de Organización Mundial de la Salud, (OMS) que va de los $9 \mathrm{~m}^{2} \mathrm{hab}^{-1}$ a los $12 \mathrm{~m}^{2} \mathrm{hab}^{-1}$.

En 2017, Pacheco \& Ávila desarrollaron una propuesta metodológica cuya área de estudio consistió en 44 parques en la ciudad de Cuenca en Ecuador, con el propósito de inventariar las especies arbóreas existentes, logrando consolidar un inventario de 2,842 árboles.

En el caso de México en el 2015, el Instituto Ciudadano de Planeación Municipal de la ciudad de Tuxtla Gutiérrez en Chiapas, en su estudio sobre la valoración de los parques urbanos ubica a estos, como "elementos vitales del espacio público" por ser lugares que propician el encuentro y desarrollo social además de tener una función ambiental como centros proveedores de oxígeno y captura de carbono.

La Asociación Nacional de Parques y Recreación de México mediante un estudio cuantitativo determinó la percepción, hábitos de uso y satisfacción que se tiene de los parques en México en el 2018. Para encontrar, que la cercanía es un elemento que influye directamente sobre la preferencia al decidir visitar o no un parque; siendo esta no mayor a 10 minutos caminando.

Retomando la técnica de fotointerpretación cartográfica, el análisis cuantitativo de Hernández Romero , Gutiérrez Cedillo, Juan Pérez, Franco Plata, \& Czerny, 2018, del 2018, ubicó, dimensionó e inventarió las áreas verdes para realizar la representación cartográfica. Adicionalmente, llevó a cabo un análisis de autocorrelación espacial encontrando un patrón de distribución disperso para la ciudad de Toluca. 
En el 2019, se evaluó y comparó la superficie de las áreas verdes urbanas de las alcaldías de Miguel Hidalgo e Iztacalco en la temporalidad de 1990 al 2015. Cabe mencionar que este estudio consideró áreas de conservación natural y áreas verdes urbanas; por lo que el índice verde estimado es mayor que el que resultara de considerar las áreas verdes urbanas exclusivamente. Los resultados arrojaron una concentración de áreas verdes en la Alcaldía Miguel Hidalgo y un índice verde aceptable, pero se identificó una proporción desigual; por lo que no se garantiza la adecuada accesibilidad a la población de estos espacios.

Otros estudios han relacionado la calidad de vida y sentido de pertenencia con el índice verde en la ciudad de Durango, utilizando métodos de análisis de imágenes satelitales y modelos multifactoriales para determinar las influencias relativas de diferentes indicadores sobre la calidad de vida, encontrándose que existe una relación proporcional entre la calidad de vida y la extensión de las áreas verdes para la zona investigada (Blancarte Siqueiros , Pérez Verdín, \& Cortes Ortiz, 2019).

A partir de estos elementos es que surge la motivación para hacer un análisis del índice verde en parques y su disponibilidad en la CDMX, enfocándose principalmente en las alcaldías de mayor densidad poblacional. A continuación, se detalla la metodología empleada en el presente estudio. Para el cálculo de la densidad poblacional, el Índice Verde y la disponibilidad.

México se ha suscrito a los objetivos de la Agenda 2030, la cual es una hoja de ruta para erradicar la pobreza, proteger al planeta y asegurar la prosperidad para todos sin comprometer los recursos para las futuras generaciones. Esta agenda cuenta con 17 objetivos de Desarrollo Sostenible los cuales cuentan con metas específicas, mismas que inciden en diversos ámbitos y sectores. En el decimoprimero Objetivo de Desarrollo Sostenible (ODS) "Ciudades y Comunidades Sostenibles", mediante siete metas se pretende afrontar la problemática existente en las ciudades con la finalidad de aprovechar los recursos, reduciendo la contaminación y la pobreza.

La meta 11.7, establece el acceso universal a las zonas verdes, pero no es la única que compete a los parques. La meta 11.1 consiste en asegurar el acceso a servicios básicos, seguros y asequibles, la meta 11.4 protege y salvaguarda el patrimonio cultural y natural y finalmente la meta 11.6 instaura la reducción del impacto ambiental negativo, enfocándose en la calidad del aire. 
Por lo que se puede apreciar, los parques no solo son un elemento insustituible del paisaje urbano; sino que también son elementos fundamentales para el camino hacia la sostenibilidad (Naciones Unidas, 2018). Asimismo, se considera que, los parques presentan tres elementos adicionales: son el espacio de convivencia para la comunidad; representan el sistema de hábitat de flora y fauna necesarios para la oxigenación de una parte de la ciudad; además es un espacio de comunión o de unión social y son referente para identificar, precisar un emplazamiento histórico y una interrelación entre sociedadnaturaleza-sociedad.

Como parte de las acciones para dar seguimiento a la agenda 2030, en agosto del 2020, se publicó la Estrategia Legislativa para la Agenda 2030, misma que fue el resultado de investigación, consultas y análisis en colaboración de la Administración Pública Federal, académicos del Instituto Tecnológico de Monterrey y la Universidad Nacional Autónoma de México y la Asociación Civil "Política y Legislación Ambiental" (POLEA) con el fin de perfilar los retos y pendientes primordiales en la incorporación a la sostenibilidad. Este documento "ofrece un diagnóstico del marco normativo federal vigente de los 17 ODS" (Estrategia Legislativa para la Agenda 2030, 2020). Para efectos del presente estudio se destaca que los compromisos de la Ley General de Asentamientos Humanos, Ordenamiento Territorial y Desarrollo Urbano no han sido cubiertos, especialmente la unificación de criterios y reglamentos a nivel municipal (Estrategia Legislativa para la Agenda 2030, 2020).

Adicionalmente, el 21 de diciembre del 2020, se publicó en el Diario Oficial de la Federación el Proyecto Oficial de La Norma de Espacios Públicos en Los Asentamientos Humanos, PROYECTO de Norma Oficial Mexicana PROY-NOM-001-SEDATU-2020, Espacios públicos en los asentamientos humanos. Que al reconocer la relevancia del espacio público para la ciudad pretende normar a este, "sentando las bases para generar redes de espacios públicos en los asentamientos humanos".

A pesar de que este proyecto de norma intenta "Homologar la terminología de los espacios públicos [para ser aplicados] en los planes o programas de Desarrollo Urbano, incluyendo su clasificación y sus componentes" (PROYECTO de Norma Oficial Mexicana PROY-NOM-001-SEDATU-2020, Espacios públicos en los asentamientos humanos, 2020, 21 diciembre), no incluye una tipología específica para parques, 
quedando subsumidos nuevamente dentro de la categoría general de las áreas verdes urbanas.

En el caso específico de la CDMX, la Ley Ambiental de Protección a la Tierra en el Distrito Federal en su última modificación del 2019, define parque como: "Las áreas verdes o espacios abiertos jardinados de uso público, ubicados dentro de suelo urbano o dentro de los límites administrativos de la zona urbana de los centros de población y poblados rurales en suelo de conservación, que contribuyen a mantener el equilibrio ecológico dentro de las demarcaciones en que se localizan, y que ofrecen fundamentalmente espacios recreativos para sus habitantes"

Los parques han sido estudiados principalmente en el contexto de las áreas verdes y son un concepto relativamente reciente, junto al concepto de ciudad, históricamente en el ámbito privado, como jardines. (Ballester Olmos \& Morata Carrasco, 2001). No obstante, los enfoques y criterios aplicados difieren sustancialmente; por lo que no es posible compararlos (Maldonado Bernabé, Chacalo Hilu, Nava Bolaños, Paredes, \& A., 2019).

Entre las múltiples funciones de los parques públicos están el esparcimiento, la biodiversidad y sus funciones ambientales. (Asociación Nacional de Parques y Recreación de México, 2020). Adicionalmente estas quedan encuadradas en tres valores fundamentales: a) Ecológico, b) Paisaje Arquitectónico y c) Socioeconómico lo que hace posible diversas actividades, por ejemplo: las recreativas, deportivas, culturales y lúdicas. Se ha hecho notar también que impactan directamente en la salud de los usuarios mitigando el estrés y mejorando la salud física - mental. (Valdés \& Foulkes, 2016), (Pope, y otros, 2018). Los parques públicos impulsan la cohesión social y potencian la biodiversidad en la ciudad (Asociación de Empresas de Gestión de Infraestructura Verde (ASEJA), Federación Española de Municipios y Provincias, Asociación Española de Parques y Jardines Públicos (AEPJP), 2019). A pesar de esto, hasta que la urbanización comienza a presentarse con aspectos negativos que el espacio verde accesible al público en general es considerado como una contraparte a las cualidades negativas de la urbanización y a tomar importancia.

La urbanización es un fenómeno de importancia fundamental para la planeación territorial y la arquitectura del paisaje. En el año 2007, se alcanzó un punto en el cual la población urbana excede a la población rural por primera vez en la historia de nuestra especie. Dada la variedad de objetivos en materias de salud, esparcimiento, acceso y transporte 
delineados en el marco de la agenda 2030 y de la sustentabilidad en la planeación urbana, se deben tomar en cuenta 3 sectores principales para tratar de mantener un balance entre ellos: ambiente, economía y sociedad (Naciones Unidas, 2018)

Dada la disparidad de objetivos a optimizar, desde hace tiempo se han utilizado herramientas tecnológicas y científicas en ayuda de la construcción de indicadores para estas tareas. Existen diferentes tipos de indicadores que pueden ser obtenidos mediante el análisis de datos convencional o mediante técnicas de procesamiento de imágenes satelitales. Algunas de las que se han utilizado para medir y aliviar efectos ambientales son: Temperaturas superficiales, análisis de los materiales superficiales, albedo superficial, cobertura fraccional de la superficie, diversos índices de vegetación, cobertura terrestre fraccional, densidad de construcción y sus cambios.

En el caso de los índices de vegetación y áreas verdes existen publicados a la fecha más de 150 índices de vegetación diferentes en la literatura científica. De los cuales solo un pequeño subconjunto ha sido validado de manera sistemática. En general, estos índices se generan para una región de la superficie terrestre utilizando el área y diferentes propiedades ópticas de la cobertura vegetal. De acuerdo con la PAOT, el índice verde se define como "un indicador de calidad de vida para habitantes de zonas urbanas. Lo establece la Organización Mundial de la Salud y recomienda de 9 a $12 \mathrm{~m}^{2}$ de AV/habitante" (PAOT 2016). Aclarando que para su determinación se ha cuantificado el total de la infraestructura verde integrada por árboles, pastos, arbustos, áreas deportivas, en suelo urbano y bosques matorrales y pastizales en suelo de conservación; mediante el procesamiento de imágenes o bases geo referenciadas de la zona de interés y se obtiene el área total cubierta por áreas verdes, este valor es referido al número de habitantes en la región de interés (PAOT 2016).

Adicionalmente, cabe resaltar que varios autores, incluyendo a la PAOT, han citado a la OMS como un parámetro que permite aplicar un criterio sobre la suficiencia recomendada de áreas verdes. Sin embargo, a pesar de haber hecho una extensa búsqueda en las publicaciones de la OMS no ha sido posible encontrar la fuente directa para validar la misma. No obstante, es importante considerar el parámetro que generalmente es considerado y aplicado como un indicador internacional del índice verde. 


\section{ESTRATEGIAS METODOLÓGICAS}

La CDMX se localiza en las siguientes coordenadas geográficas: Longitud 99²1'53.642” W a $98^{\circ} 56^{\prime 2} 25.08^{\prime \prime}$ W; Latitud 19²7'23.04” N a 19³0“54.36” N. (Instituto Nacional de Estadística y Geografía, 2020). De acuerdo con el último censo realizado por el INEGI en el 2020; la CDMX cuenta con una población total de 9,209,944 habitantes; donde el $52.2 \%$ son mujeres y el $47.8 \%$ hombres. El total de viviendas particulares reportado en 2020 equivale a 3,035,125, de estas el 90.8\% estaban habitadas, el 6.8\% se encontró deshabitado, siendo el resto de uso temporal. En cuanto a la cobertura de servicios urbanos, las viviendas particulares reportan que el 99.9\% cuenta con servicio de energía eléctrica, el $98.9 \%$ cuenta con agua entubada y el servicio de drenaje corresponde al 99.7\%. (INEGI, 2021).

A efecto de poder delimitar el área de estudio, se consideró como aspecto prioritario aquellas alcaldías con mayor concentración poblacional, basándonos en datos de INEGI 2020, así como su dinámica poblacional en el periodo 2017 y 2020. Datos de densidad de población fueron obtenidos.

Los análisis cuantitativos fueron desarrollados en una computadora portátil marca Asus con el sistema operativo Windows 8.1, 8 GB de RAM y un procesador Intel i7 a $2.4 \mathrm{GHz}$. Se utilizó el software Mapa Digital V 6.3.0 de INEGI para los cálculos demográficos y de índice verde. La edición cartográfica fue realizada en el programa QGIS en la versión 2.18 "Las Palmas" (QGIS, 2016). Los datos abiertos de la Secretaría del Medio Ambiente (SEDEMA, 2017) y las proyecciones de la población de los municipios de México (CONAPO, 2016) fueron los utilizados.

El primer paso consistió en analizar los metadatos y la estructura de los atributos de cada una de las capas a utilizar para verificar la calidad de datos, homologar proyecciones y unidades; después de este análisis se requirió de un preprocesamiento de las bases de datos, en donde se homologaron las proyecciones cartográficas al Sistema de Coordenadas International Terrestrial Reference Frame 2008 y al Sistema de Proyección Universal Transverse Mercator de cada capa vectorial. A diferencia de estudios anteriores, no se utilizaron imágenes satelitales para la determinación del Índice Verde, ya que estas no permiten de manera simple la caracterización de las áreas dependiendo de su tipología. 
Mediante el uso del Mapa Digital de México V6.3.01 se realizaron procesos de geoprocesamiento de las bases de datos del INEGI, (INEGI, 2020) se determinó el índice verde de áreas verdes en parques entre los habitantes por alcaldía seleccionada para los años 2017 y 2020. Es notable mencionar que para este estudio se consideraron únicamente las áreas verdes correspondientes a parques en suelo urbano de la CDMX.

Es importante resaltar que solo se cuenta con el levantamiento de áreas verdes del 2017; sin embargo, la actualización de la información de la población aportó más información para ampliar el panorama actual.

Con el propósito de estandarizar la geometría y la tabla de atributos de la capa vectorial correspondiente a la base de datos del inventario de áreas verdes del 2017(Comité Consultivo Nacional de Normalización de Ordenamiento Territorial y Desarrollo Urbano, 2020), se procedió a analizar cada polígono georreferenciado dentro del área de estudio con el apoyo de Google Maps.

Se retoma y adaptan los criterios del anexo metodológico aplicados por el Centro UC Políticas Públicas, 2019 aplicando los siguientes criterios sin modificar la vectorización original: Se unificaron aquellos grupos de polígonos que en conjunto corresponden a un solo parque; Se consideró como un solo elemento a aquellos polígonos de parques que al ser desplantados sobre la capa de infraestructura vial se dividían en 2 o más polígonos y se decidió unificar aquellos polígonos divididos por una vialidad, ya que se determinó que constituyen una misma área de servicio debido a su proximidad.

Una vez aplicados estos criterios, se editó la tabla de atributos de la capa de parques calculando las superficies de cada elemento y dado que muchos de ellos no están identificados en la base con un nombre en particular, se renombraron bajo la nomenclatura Parque_SN_\#, otorgándose una numeración consecutiva a cada elemento renombrado. Finalmente se realizó la edición cartográfica por alcaldía con el software QGIS.

\section{RESULTADOS Y DISCUSIÓN}

Derivado del análisis de la información poblacional con datos del Censo 2020 de Población y Vivienda, se determinaron las alcaldías con el mayor valor de concentración demográfica, delimitando con ello nuestras zonas de interés quedando enmarcada en las de Iztacalco, Iztapalapa, Benito Juárez y Cuauhtémoc, obteniendo para cada caso la cartografía respectiva. 
En la figura 1, se muestra la distribución de los distintos parques que fueron fusionados mediante la aplicación de los tres criterios mencionados en la metodología (color naranja) así como los parques simples en color verde, adicionalmente el color gris representa la traza urbana a nivel manzana de cada alcaldía.
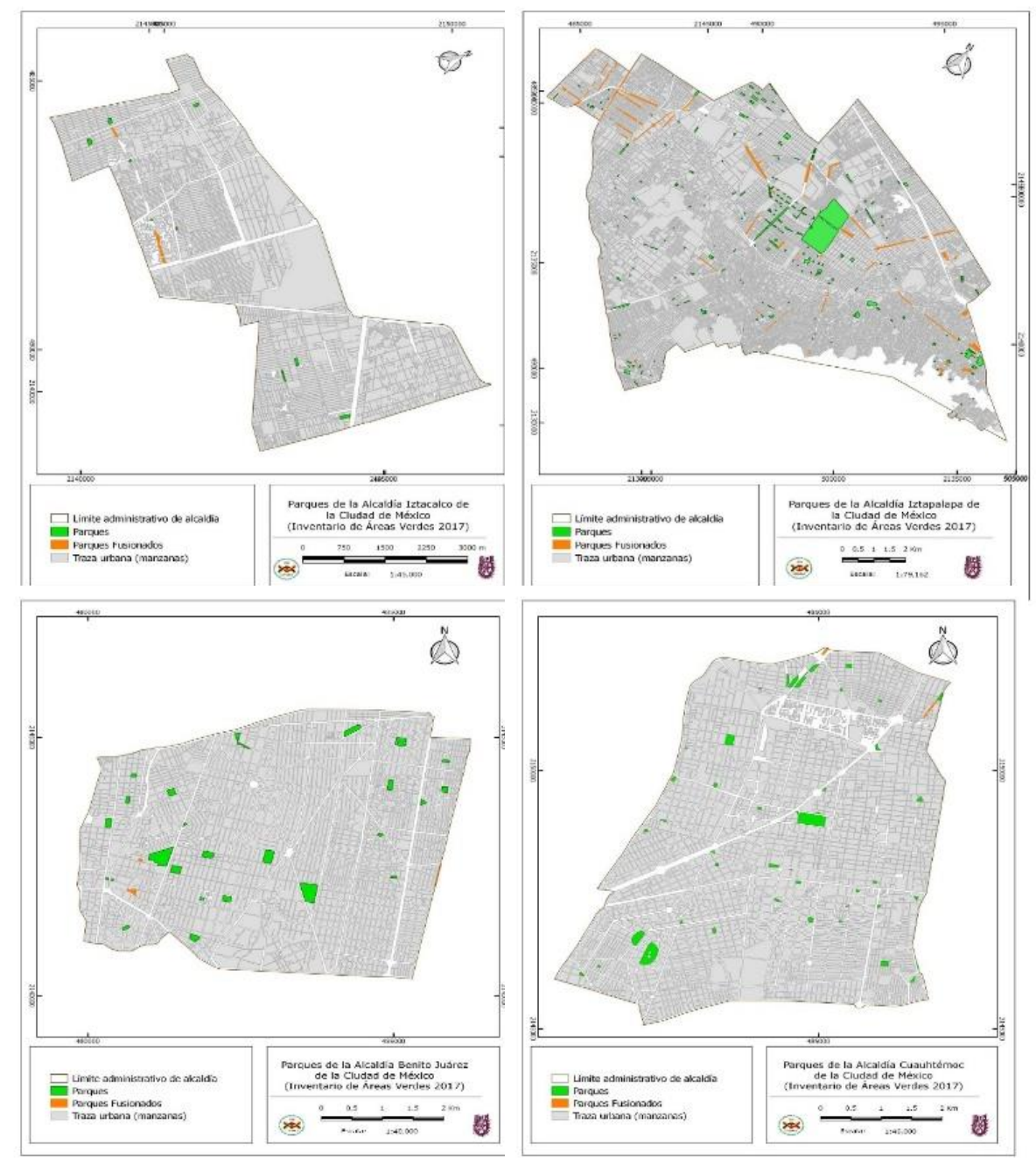

Figura 1: Distribución de los distintos parques públicos en las cuatro alcaldías seleccionadas: Iztacalco e Iztapalapa, Benito Juárez y Cuauhtémoc (2017). Fuente: Elaboración propia con información de SEDEMA e INEGI

Los parámetros evaluados por alcaldía, obtenidos del geoprocesamiento se mencionan en la Tabla 1 siguiente: 
Tabla 1. Parámetros evaluados por alcaldía en la zona de estudio

\begin{tabular}{|c|c|c|c|c|}
\hline \multirow{2}{*}{ Parámetros } & \multicolumn{4}{|c|}{ Alcaldías } \\
\hline & Iztacalco & Iztapalapa & Benito Juárez & Cuauhtémoc \\
\hline $\begin{array}{c}\text { Población proyectada } 2017 \\
\text { CONAPO } \\
\text { (hab) }\end{array}$ & 395,939 & $1,837,496$ & 422,882 & 545,675 \\
\hline $\begin{array}{l}\text { Población Censo } 2020 \text { INEGI } \\
\text { (hab) }\end{array}$ & 404,695 & $1,835,486$ & 434,153 & 414,470 \\
\hline $\begin{array}{l}\text { Superficie total } \\
\left(\mathrm{Km}^{2}\right)\end{array}$ & 23.1 & 113.17 & 26.7 & 32.52 \\
\hline $\begin{array}{l}\text { Densidad de población al } 2017 \\
\qquad\left(\mathrm{hab} \mathrm{Km}^{-2}\right)\end{array}$ & $17,140.21$ & $16,236.59$ & $15,838.27$ & $16,779.67$ \\
\hline $\begin{array}{c}\text { Densidad de población al } 2020 \\
\left(\mathrm{hab} \mathrm{Km}^{-2}\right)\end{array}$ & $17,519.26$ & $16,218.83$ & $16,260.41$ & $12,745.07$ \\
\hline $\begin{array}{l}\text { Superficie total de áreas verdes en } \\
\text { parques }\left(\mathrm{Km}^{2}\right)\end{array}$ & 0.08 & 3.02 & 0.44 & 0.37 \\
\hline $\begin{array}{l}\text { Índice verde de áreas verdes en parques } \\
\text { entre los habitantes } 2017 \\
\qquad\left(\mathrm{~m}^{2} \mathrm{hab}^{-1}\right)\end{array}$ & 0.2 & 1.64 & 1.04 & 0.68 \\
\hline $\begin{array}{l}\text { Índice verde de áreas verdes en parques } \\
\text { entre los habitantes } 2020 \\
\qquad\left(\mathrm{~m}^{2} \mathrm{hab}^{-1}\right)\end{array}$ & 0.19 & 1.65 & 1.01 & 0.9 \\
\hline $\begin{array}{l}\text { Porcentaje territorial ocupado por áreas } \\
\text { verdes en parques }\end{array}$ & $0.34 \%$ & $2.67 \%$ & $1.65 \%$ & $1.15 \%$ \\
\hline
\end{tabular}

Fuente: Elaboración propia con información de CONAPO e INEGI

Para el periodo 2017-2020, el valor de densidad de población más representativo a la baja se da en la alcaldía de Cuauhtémoc pasando de 16,779 hab km-2 en 2017 a 12,745 hab $\mathrm{km}^{2}$ en 2020, que es una disminución del $24 \%$, dejando de ser, con ello, una de las más densamente pobladas de la CDMX.

En las Figuras 2 y 3 correspondientes a los mapas de densidad de población de la CDMX para los años del 2017 y 2020 respectivamente, se puede apreciar el cambio en la densidad de población entre las diferentes alcaldías entre los años 2017 y 2020. Es notable que la población se ha concentrado aún más en 3 alcaldías, por lo que estas demandarán mayor 
cantidad y calidad de servicios urbanos incluidos los ecosistémicos, ambientales y sociales que los parques aportan a los residentes de esta zona.

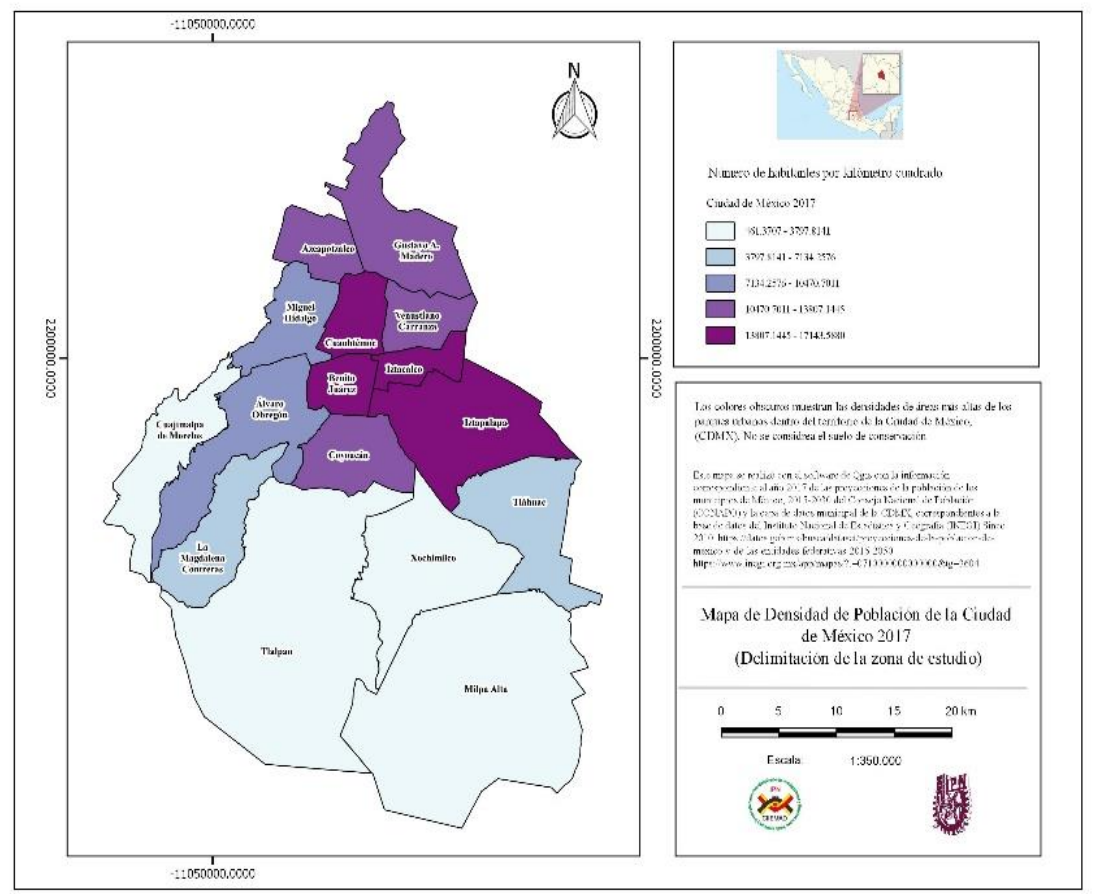

Figura 2: Densidad de población para la CDMX 2017.

Fuente: Elaboración propia con Información del INEGI y la CONAPO

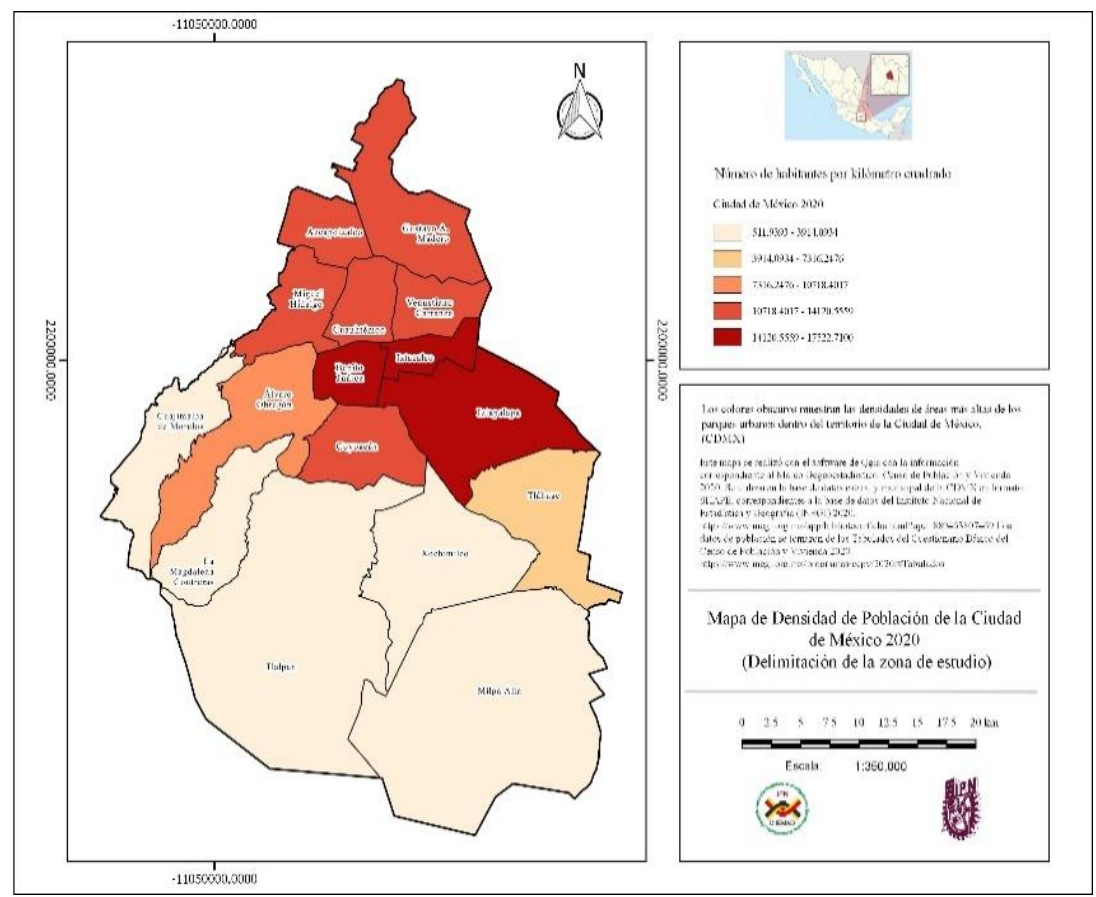

Figura 3: Densidad de población para la CDMX (2020). 
En la Figura 4, se presenta el número de parques por alcaldía, de acuerdo con el conteo realizado después de haber aplicado los criterios de unificación.

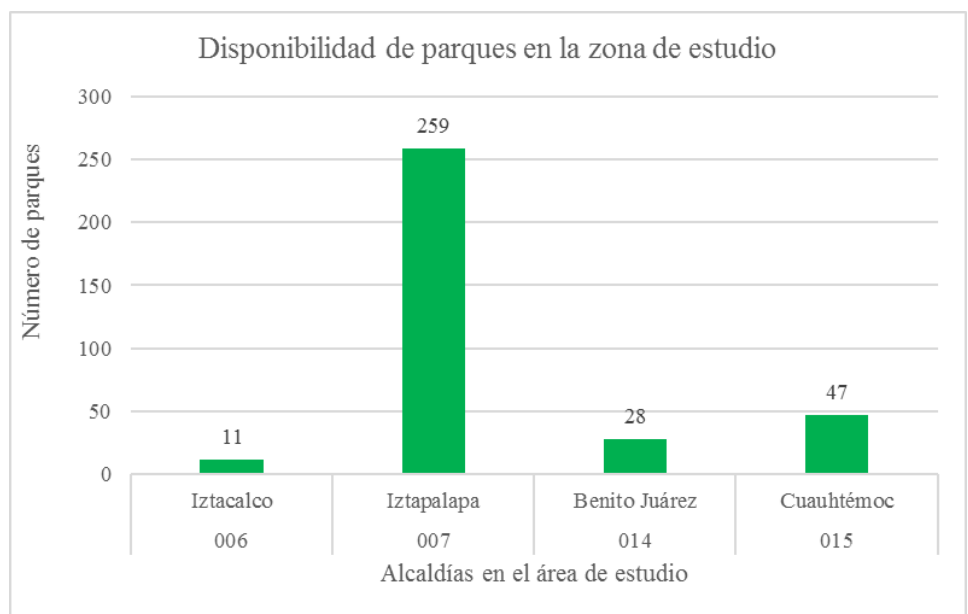

Figura 4: Disponibilidad de parques en la zona de estudio, 2017.

Fuente: Elaboración propia con información de SEDEMA e INEGI.

Como se puede apreciar, la alcaldía de Iztapalapa es la que mayor disponibilidad de parques tiene (259), le sigue Cuauhtémoc con 47 y Benito Juárez con 28, destacando Iztacalco con apenas 11 a pesar de ser la alcaldía con mayor densidad de población.

Por otra parte, la figura 5, muestra el índice verde de áreas verdes por alcaldía, destacando nuevamente en este rubro la alcaldía de Iztapalapa con un valor de $1.64 \mathrm{~m}^{2} \mathrm{hab}^{-1}$. La alcaldía Benito Juárez le sigue con un valor de1.04 $\mathrm{m}^{2} \mathrm{hab}^{-1}$, estando al final de este indicador la alcaldía de Iztacalco con $0.2 \mathrm{~m}^{2} \mathrm{hab}^{-1}$.

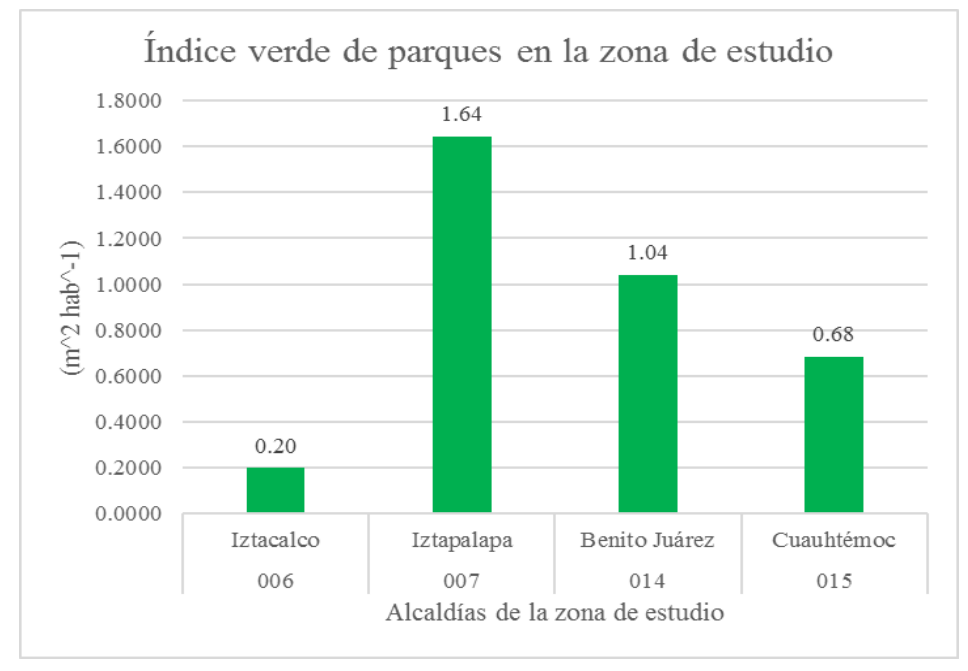


Figura 5: Índice verde de áreas verdes en parques entre los habitantes por alcaldía

2017. Fuente: Elaboración propia con información de SEDEMA e INEGI.

Lo anterior refleja la enorme necesidad que existe de abrir espacios verdes recreativos principalmente orientados a las demarcaciones de Iztacalco y Benito Juárez puesto que es un área en donde cada vez hay mayor concentración de habitantes. La alcaldía Cuauhtémoc de igual manera requiere de reforzar la apertura de nuevos espacios verdes a efecto de equilibrar su densidad de población con su Índice Verde en parques.

\section{CONSIDERACIONES FINALES}

La determinación del índice verde en parques evidenció la necesidad de espacios verdes que permitan no sólo un espacio recreativo como normalmente se etiqueta a los parques, sino que son espacios que propician la interacción con la naturaleza rompiendo con el esquema de elementos antropomorfizados del paisaje urbano. Son elementos del espacio público que no sólo aportan valor escénico, sino que también promueven inclusión y cohesión social, impactando directamente en el medio ambiente y en la calidad de vida de los residentes urbanos.

Además, deben considerarse en la planeación urbana; ya que su existencia y accesibilidad impulsa el desarrollo sustentable de acuerdo con los objetivos de la agenda 2030. Sin embargo, el índice verde que normalmente se estima, es calculado con el total de las áreas verdes, lo que resta importancia a la necesidad que el Levantamiento de Áreas Verdes del 2017 plantea.

Hace falta estandarizar criterios de clasificación de los diferentes elementos que conforman el total de la infraestructura verde de la CDMX; ya que hay dos predios en la Alcaldía de Benito Juárez que, a pesar de ser de acceso público, quedan fuera de la jurisdicción estatal al ser atrios de Iglesias.

El avance tecnológico ha permitido desarrollar diversas bases de datos de información en los Sistemas de Información Geográficas mismas que son herramientas fundamentales para la realización de los Planes de Desarrollo Urbano. No obstante, se requiere homogenizar criterios para la creación, integración y uso de esta información para que sea veraz y funcional. Adicionalmente, dado que el inventario utilizado es del año 2017, es deseable una actualización del inventario de parques disponible en formato abierto.

Principalmente en la alcaldía de Iztapalapa, es visible que varios camellones de la infraestructura vial funcionan como parques. Por lo que analizar las características de los 
camellones, permitiría explotarlos para incrementar el número de parques creando una red de parques lineales que se conectara con los elementos poligonales. De esta forma se aumentaría la disponibilidad, el índice verde de parques y la accesibilidad a estos espacios.

\section{AGRADECIMIENTOS}

La primer autora agradece al Consejo Nacional de Ciencia y Tecnología (CONACyT) por la beca doctoral con número de registro 301673 otorgada, para cursar el Doctorado en Ciencias en Conservación del Patrimonio Paisajístico en el Centro Interdisciplinario de Investigaciones y Estudios sobre Medio Ambiente y Desarrollo (CIIEMAD) del Instituto Politécnico Nacional, este estudio deriva del tema de tesis doctoral "Análisis de la distribución territorial de los parques en el paisaje urbano de la Ciudad de México"; al INEGI y SEDEMA por el acceso libre a sus bases de datos abiertos; A los Ejecutivos de Cuenta Roberto Sánchez Pérez y Edgar Adrián Martínez Haro de INEGI por su inestimable apoyo en la verificación de información y procedimientos; al doctor Otto Hahn por su apoyo editorial

\section{REFERENCIAS}

Asociación de Empresas de Gestión de Infraestructura Verde (ASEJA), Federación Española de Municipios y Provincias, Asociación Española de Parques y Jardines Públicos (AEPJP). (2019). Guía Divulgativa de la Infraestructura Verde Municipal. Obtenido de http://redbiodiversidad.es/gestión-deinfraestructuras-verdes (GUIA-DIVULGATIVA-DEFINITIVA, P. 2: 612)

Asociación Nacional de Parques y Recreación de México ANPR México. (2018).

Viviendo los parques Usos y Costumbres de los Mexicanos. Ciudad de México: ANPR México.

Asociación Nacional de Parques y Recreación de México. (Julio de 2020). ¿Son importantes los parques Urbanos? Guía infográfica completa sobre sus beneficios y nondades. México, México: ANPR, México.

Ballester Olmos, J. F., \& Morata Carrasco, A. A. (2001). Normas para la clasificación de los espacios verdes. Valencia: Universidad Politécnica de Valencia. 
Blancarte Siqueiros , R. H., Pérez Verdín, G., \& Cortes Ortiz, A. (19 de octubre de 2019). LThe relationship between quality of life, sense of belonging, and green spaces in urban environments in the city of Durango, Mexico. Revista Chapingo Serie Ciencias Forestales y del Ambiente, 97-111. doi:10.5154/r.rchscfa.2019.03.023

Briz de Felipe, T., \& Felipe, B. I. (2017). A methodological approach for urban green areas: a case study in Madrid. Revista Chapingo Serie Ciencias Forestales y del Ambiente, 23(2), 315-328. doi:http://dx.doi.org/10.5154/r.rchscfa.2016.03.012

Centro UC Políticas Públicas. (2019). Desafios en la accesibilidad áreas verdes enla ciudad y posibles vias de solucion en el marco de la ley de aportes. Colombia. Obtenido de https://politicaspublicas.uc.cl/wpcontent//uploads/2019/02/2019.01.30_REPORTE-DEL-ESTUDIO.pdf

CONAPO. (2016). Proyecciones de la Población de México y de las Entidafes Federativas 2015-2050. Obtenido de : https://datos.gob.mx/busca/dataset/proyecciones-de-la-poblacion-de-mexico-yde-las-entidades-federativas-2016-2050/resource/aab5b82a-782a-4caa-9390a0afcbbe393c

Estrategia Legislativa para la Agenda 2030. (24 de agosto de 2020). México. Obtenido de https://www.gob.mx/cms/uploads/attachment/file/573730/Estrategia_Legislativa _para_la_Agenda_2030_VF_comp.pdf

Galfioni, M. d., Degioanni, A. J., \& Maldonado , G. I. (2017). Disponibilidad y accesibilidad a los espacios verdes públicos en la ciudad de Río Cuarto (Argentina). Memorias y boletines de la Universidad del Azuay, XVI. Obtenido de http://revistas.uazuay.edu.ec/index.php/memorias/article/view/71

Gobierno de la Ciudad de México. (noviembre de 2019). Programa de Gobierno 2019 2014. Obtenido de https://plazapublica.cdmx.gob.mx/uploads/decidim/attachment/file/12/plan_gob _nov_digital.pdf 
Gómez , N. J., \& Velázquez, G. A. (2018). Asociación entre los espacios verdes públicos y la calidad de vida en el municipio de Santa Fe, Argentina. Cuadernos de Geografía, 27(1). doi:https://doi.org/10.15446/rcdg.v27n1.58740

Hernández Romero , J. C., Gutiérrez Cedillo, J., Juan Pérez, J. I., Franco Plata, R., \& Czerny, M. (2018). Distribución de áreas verdes y bienestar socio ambiental. Un estudio en la Ciudad de Toluca, México. Revista Latinoamerivcana el AMbiente y las Ciencias, 919 - 942. Obtenido de http://cmas.siu.buap.mx/portal_pprd/work/sites/rlac/resources/LocalContent/86/ 2/9(21)-63.pdf

INEGI (Instituto Nacional de Estadistica y Geografía). (2017). Marco Geoestadístico 2010. Obtenido de https://www.inegi.org.mx/app/biblioteca/ficha.html?upc=889463142683

INEGI (Instituto Nacional de Estadistica y Geografía). (2019). Mapa Digital de México . Obtenido de https://www.inegi.org.mx/temas/mapadigital/

INEGI (Instituto Nacional de Estadistica y Geografía). (2020). Marco Geoestadístico 2020. Obtenido de https://www.inegi.org.mx/app/biblioteca/ficha.html?upc=889463807469\&fbclid =IwAR3VKpzE5wi5wjaFaAqpm9xOirxLBjSdYcAaegmMwzKxwfKOHpRe_p 4-7B4

INEGI (Instituto Nacional de Estadistica y Geografía). (2021). Comunicado de prensa 98/21. Recuperado el 23 de marzo de 2021, de https://www.inegi.org.mx/contenidos/saladeprensa/boletines/2021/EstSociodem o/ResultCenso2020_CdMx.pdf

Instituto Ciudadano de Planeación Municipal. (2015). Valoración de los parques Urbanos. La ruta hacia una Tuxtla sustentable. Tuxtla.

Ley Ambiental de Protección a la Tierra. (8 de septiembre de 2019, 25 de junio). Gaceta Oficial de la Ciudad de México. Obtenido de Procuraduría Ambiental y del Ordenamiento Territorial de la CDMX: 
hhttp://www.data.consejeria.cdmx.gob.mx/images/leyes/leyes/LEY_AMBIENT AL_DE_PROTECCION_A_LA_TIERRA_DF.pdf

Maldonado Bernabé, G., Chacalo Hilu, I., Nava Bolaños, R., Paredes, M., \& A., Z. H. (Julio de 2019). Cambios en la superficie de áreas verdes urbanas en dos alcaldías de la ciudad de méxico entre 1990 - 2015. (I. P. Nacional, Ed.) Polibotánica(48), 205 - 230. doi:10.18387/polibotanica.48.15

Naciones Unidas. (2018). La Agenda 2030 y los Objetivos de Desarrollo Sostenible: una oportunidad para América Latina y el Caribe. Santiago: Naciones Unidas. Obtenido de https://repositorio.cepal.org/bitstream/handle/11362/40155/24/S1801141_es.pdf

Pacheco, D., \& Ávila, L. (2017). Inventario de Parques y jardines de la ciudad de Cuenca con UAV y smartphones. Infraestructura de datos espaciales (págs. 173 - 179). Cuenca: Universidad del Azuay. Obtenido de http://revistas.uazuay.edu.ec/index.php/memorias/article/download/61/55/

PAOT (Procuraduría Ambiental y del Ordenamiento Territorial de la CDMX). (2016). Datos del íncide verde en colonias a afectar por construcción del Deprimido Vehicular Insurgentes- Mixcoac. Obtenido de http://centro.paot.org.mx/documentos/paot/estudios/av_mixcoac.pdf

PAOT (Procuraduría Ambiental y del Ordenamiento Territorial de la CDMX). (2010). Presente y futuro de las áreas verdes y del arbolado de la ciudad de México. cdmx: PAOT.

PROYECTO de Norma Oficial Mexicana PROY-NOM-001-SEDATU-2020, Espacios públicos en los asentamientos humanos. (2020, 21 diciembre). Diario Oficial de la Federación.

Pope, D., Tisdall, R., Middleton, J., Verma, A., van Ameijden, E., Birt, C., . . Bruce, N. (1 de febrero de 2018). Quality of and acces to green space in relation to psycological distrss:results from a populatio-based cross-sectional study as part of the EURO-URHIS 2 project. Associations of green space with psychological distress, 1(28), 35-38. doi:doi: 10.1093/eurpub/ckv094. 
QGIS.ORG.2016. (s.f.). QGIS Geographic Information System. OGIS Association. Obtenido de http://www.qgis.org/

Reyes Paecke, S., \& Figueroa, I. (2010). Distribución y accesibilidad de las áreas verds en Santiago de Chile. EURE. Revista laninoamericana de estudios urbano regionales. doi:10.4067/S0250-71612010000300004

SEDEMA (Secretaría de Medio Ambiente). (2017). Inventario de áreas verdes. Una colaboración interinstitucional entre el gobierno de la CDMX bajo la coordinación de la Secretaría de Medio Ambiente (SEDEMA) con la Universidad Nacional Autónoma de México (Instituto de Geografía). Metadatos solicitados via portal de trasparencia a S. Obtenido de https://datos.cdmx.gob.mx/explore/dataset/cdmx_areas_verdes_2017/export/?loc ation=10,19.37117,-99.13727 23 de octubre 2019 último acceso

Valdés, P., \& Foulkes, M. D. (2016). La infraestructura verde y su papael en el desarrollo regional. Aplicación a los ejes recreativoc y culturales de resistencia y su área metropolitana. Cuaderno Urbano. Espacio, Cultura, Sociedad, 20(20), 47-50. Obtenido de https://dialnet.unirioja.es/servlet/articulo?codigo=5962118 